Article type : Review

\title{
Current issues with luminal subtype classification in terms of prediction of benefit from endocrine therapy in early breast cancer
}

Running title: Luminal breast cancer and benefit of endocrine therapy

Lutfi Alfarsi, ${ }^{1}$ Simon Johnston, ${ }^{1}$ Dong-Xu Liu, ${ }^{3}$ Emad Rakha ${ }^{1,2}$ and Andrew Green ${ }^{1}$

${ }^{1}$ Academic Pathology, Division of Cancer and Stem Cells, School of Medicine, University of Nottingham, Nottingham City Hospital, Hucknall Road, Nottingham NG5 1PB; ${ }^{2}$ Cellular Pathology, Nottingham University Hospitals NHS Trust, Hucknall Road, Nottingham NG5 1PB; ${ }^{3}$ The Centre for Biomedical and Chemical Sciences, School of Science, Faculty of Health and Environmental Sciences, Auckland University of Technology, Auckland 1010, New Zealand.

\section{Correspondence:}

Dr Andrew R. Green. Academic Pathology, Division of Cancer and Stem Cells, School of Medicine, University of Nottingham, Nottingham City Hospital, Hucknall Road, Nottingham NG5 1PB

Tel: (44) 1158231407

Email: andrew.green@nottingham.ac.uk

Conflict of interest: The authors confirm that they do not have any conflict of interest.

Author contributions: All authors have contributed to the drafting, critical revision and final approval of the version submitted.
Abstract
Endocrine therapy for oestrogen receptor-positive $(\mathrm{ER}+)$ breast cancer $(\mathrm{BC})$ is arguably the most successful targeted cancer therapy to date. Nevertheless, resistance to endocrine therapy still occurs in a significant proportion of patients, limiting its clinical utility. ER+ or luminal This article has been accepted for publication and undergone full peer review but has not been through the copyediting, typesetting, pagination and proofreading process, which may lead to differences between this version and the Version of Record. Please cite this article as doi: $10.1111 /$ his. 13523
This article is protected by copyright. All rights reserved. 
$\mathrm{BC}$, which represents around three quarters of all breast malignancies, are biologically heterogeneous with no distinct, clinically defined sub-classes able to predict the benefit of endocrine therapy in early settings. To improve patient outcomes, there is a clear need for improved understanding of the biology of the luminal $\mathrm{BC}$, with subsequent translation into more effective methods of diagnosis to identify potential predictive biomarkers for endocrine therapy. This review summarises current knowledge of factors predictive of benefit of endocrine therapy, and discusses why molecular classification systems of $\mathrm{BC}$ have yet to be translated into the clinic.

Keywords: breast cancer, luminal, endocrine therapy response, resistance, oestrogen receptor, predictive biomarker

\section{Introduction}

Oestrogen receptor (ER) is the driving transcription factor in up to three-quarters of all $\mathrm{BC}$ and its protein expression by immunohistochemistry classifies patients as either having ER+ or ER-negative (ER-) disease. Endocrine therapy is one of the most effective and wellestablished targeted anti-cancer treatments for ER+ BC. However, despite its undisputed efficacy, up to one third of patients will relapse after treatment for early stage disease, whilst in the advanced setting, all will eventually progress and become resistant [1]. It is therefore desirable to be able to predict, at an early stage of treatment, which ER+ patients will benefit from endocrine therapy, and which are likely to develop resistance, so that alternative or additional therapies can be offered to prevent relapse and reduce $\mathrm{BC}$ specific mortality. The identification of biomarkers to predict endocrine therapy benefit in addition to ER status is therefore of crucial importance in stratifying ER+ patients for targeted therapy. During the past two decades, the molecular era has promised much with respect to prediction of benefit 
from endocrine therapy in early $\mathrm{BC}$, but clinicians remain unable to predict which of their patients will benefit from endocrine therapy.

ER and progesterone receptor (PR) measurements are currently used for both clinical diagnosis to classify $\mathrm{BC}$ patients and as a guide to endocrine therapy [2]. Although the absence of ER expression is well known predictor of lack of hormonal therapy effect, the response of ER+ tumours to hormone therapy is heterogeneous. The role of PR measurement to predict benefit to endocrine therapy remains controversial. Some studies suggests its measurement lacks clinical value in $\mathrm{BC}$ management [3], while other reports considered that $\mathrm{PR}$ expression is a positive prognostic factor in $\mathrm{ER}+\mathrm{BC}[4,5]$. There is therefore a clear unmet clinical need for further biomarkers, beyond ER and PR, to help in predicting the benefit from endocrine therapy.

Global gene expression profiling (GEP) studies of BC have identified a distinct molecular subtype called 'luminal-like'. The current accepted molecular classification of luminal subtype divides them into two subgroups: luminal A and luminal B [6]. Luminal B disease demonstrates increased expression of proliferation-related genes which make it more aggressive in its clinical behaviour [7]. This subtype has a higher recurrence rate and lower survival rates after relapse [8], and is considered as a more aggressive subtype of ER+ disease, while luminal-A disease tends to result in better clinical outcomes [7]. However, such molecular classification is currently not routinely used in the clinic to guide endocrine therapy.

Thus, there remains an urgent need to identify biomarkers, singularly or in combination, and clinically relevant subclasses within $\mathrm{ER}+\mathrm{BC}$ to refine classification of luminal tumours, particularly with a view to predicting the benefit from the endocrine therapy in early BC. In this article, we provide a comprehensive review of the current modalities with potential 
applicability in predicting benefit from endocrine therapy. The article focusses on why molecular classification systems have yet to be translated successfully into the clinic for this purpose, and how the field can move forward in order to improve patient outcomes.

\section{Endocrine therapy}

Endocrine therapy is a well-established standard treatment for ER+ BC patients. Classes of endocrine therapy include selective ER modulators (SERMs) (e.g. tamoxifen), aromatase inhibitors (AI) (e.g. anastrozole, exemestane and letrozole) and selective ER down-regulators (SERDs) (e.g. fulvestrant). The key clinical questions for those with ER+ BC are 1) which endocrine therapy should be offered and 2) what is the role of biomarkers in selecting patients for the most effective therapy option?

\section{Mechanisms of action}

Current endocrine therapy works either by blocking or lowering the action of ER, or inhibiting peripheral synthesis of oestrogen from its precursors in the circulation. Tamoxifen, a SERM, is non-steroidal synthetic agent that exhibits tissue-specific ER agonist or antagonist activity. The mechanism of action of tamoxifen involves a competitive inhibition of oestrogen binding to the ER via its ligand-binding domain. This induces a conformational change in the nuclear receptor. In ER+ breast tumours, binding of tamoxifen to the ER results in inhibition of oestrogen dependent gene transcription, cell proliferation and tumour growth

[1]. Tamoxifen causes apoptosis and cell cycle arrest in G0/G1 phase via modulating growth factors. However, tamoxifen also exerts an agonist effect on the endometrium, thus increasing the risk of developing endometrial cancer as a side effect of BC treatment [1]. 
AIs reduce oestrogen levels by blocking the peripheral conversion of androgen to oestrogens catalysed by the aromatase enzyme. They are classified by their mechanisms of action into two types: steroidal agents such as exemestane, which irreversibly inactivates the enzyme, and non-steroidal such as anastrozole and letrozole, which inhibit aromatase through reversible binding [9]. AIs are recommended above tamoxifen for postmenopausal women with ER+ BC [10].

Fulvestrant, a SERD, is known as a "pure" anti-oestrogen as it does not have any agonist activity in other tissue contexts. It binds competitively to ER, inhibits its dimerisation and ultimately results in downregulation of ER expression [11]. Although fulvestrant is not currently approved by the National Institute for Health and Excellent Care (NICE) in the UK for the treatment of ER+ BC, it is widely used in the advanced setting and is approved by the US Food and Drug Administration (FDA) and European Medicines Agency (EMA) [12, 13].

\section{Success}

Tamoxifen is considered the gold standard endocrine therapy for premenopausal women with early and advanced ER+ BC, and post-menopausal women who have contra-indications to AIs [10, 14]. The 2011 meta-analyses from the Early Breast Cancer Trialists' Collaborative Group (EBCTCG) showed that, for ER+ patients, 5 years treatment with tamoxifen reduced the risk of $\mathrm{BC}$ recurrence and mortality by $39 \%$ and $30 \%$, respectively [15]. A further reduction of the recurrence risk in $\mathrm{ER}+\mathrm{BC}$ can be achieved by extending the duration of tamoxifen treatment to 10 years [16]. However, whilst there has been a significant fall in mortality rates with the use of tamoxifen, a third of patients experience resistance to tamoxifen. Resistance is classified as either primary (relapse within the first 2 years of 
adjuvant endocrine therapy) or secondary (relapse after 2 years on endocrine therapy, or within 12 months of completing adjuvant endocrine therapy) [17].

In postmenopausal women with $\mathrm{ER}+\mathrm{BC}$, treatment with $\mathrm{AIs}$ is marginally superior to tamoxifen in efficacy both in terms of the risk of recurrence and overall survival $[10,18]$. Recently, it has been reported that in premenopausal women with $\mathrm{ER}+\mathrm{BC}$, clinical outcomes for patients with high recurrence risk may be improved by treatment with AIs and ovarian function suppression compared to tamoxifen alone [19].

\section{Cyclin-dependent kinases 4 and 6 inhibitors}

Cyclin-dependent kinases 4 and $6(\mathrm{CDK} 4 / 6)$ are important drivers of the cell cycle and are required for the initiation and progression of various tumours [20, 21]. Inhibitors of CDK4/6 lead to cell cycle arrest in the G1 phase by preventing phosphorylation of retinoblastoma protein, and thereby prevent tumour progression [22]. Currently, there are three selective inhibitors of CDK4/6 (palbociclib, abemaciclib and ribociclib) have been tested in clinical BC trials. Palbociclib has been approved by the FDA and EMA, in combination with an AI as endocrine based therapy in postmenopausal women with ER+/HER2-negative advanced or metastatic BC $[23,24]$. Ribociclib has also been approved by the FDA, as initial treatment for postmenopausal women with advanced ER+/HER2-negative BC [24].

In the near future, the role of CDK4/6 inhibitors is likely to expand into the setting of early BC. For example, abemaciclib is currently being explored in combination with standard endocrine therapy for patients at higher risk of relapse [25].

This article is protected by copyright. All rights reserved. 


\section{ER and PR measurement}

The determination of ER protein expression has been used in the clinic for over 30 years to identify patients likely to benefit from endocrine therapy. Despite this, ER positivity is insufficient to predict whether an individual patient with early breast cancer is likely to experience disease relapse.

In addition to ER, there is some additional predictive benefit in measuring progesterone receptor (PR) expression, as recommended by the Royal College of Pathologists [2]. Immunohistochemistry (IHC) remains the most commonly used diagnostic technique for determination of ER and PR status as it is relatively quick, cheap and can be performed on formalin-fixed paraffin embedded (FFPE) tissue. Although the St. Gallen Guidelines recommended IHC for use in clinical practice in 2013 [14], there is no gold standard assay available for determination of ER and PR status by IHC with regard to the prediction of benefit from endocrine therapy. Until the recent decade, ER positivity was defined in routine clinical practice using a cut-off value for ER nuclear staining of $10 \%$. By this criterion, patients with $\geq 10 \%$ nuclear staining of ER were deemed eligible for endocrine therapy [26].

The American Society of Clinical Oncology (ASCO) Reported Guidelines for ER and PR IHC assays was an attempt to make the benefit of endocrine therapy available to the widest range of patients. ASCO recommends $1 \%$ as a universal cut-off point to distinguish between ER positivity and negativity to help determine likelihood of patients responding to endocrine therapy. Thus, they considered " $1 \%$ or more of cells staining for ER" as the cut-off for a specimen to be considered positive. Tumours with less than $1 \%$ of cells staining for ER are considered ER negative based on data that patients with such tumours do not receive meaningful benefit from endocrine therapy [27]. However, lowering the threshold for ER 
positivity increases the proportion of patients in whom endocrine therapy is indicated. This has raised uncomfortable questions about the low ER+ (1-9\%) tumours.

Subsequent studies have confirmed the suspicion that a significant proportion of these low$\mathrm{ER}+$ are in fact non-luminal tumours. It is therefore suggested that the most expeditious therapeutic approach may be to use both endocrine therapy and chemotherapy in this subset of patients $[28,29]$. It is reported that patients with low ER tumours $(1-9 \%)$, in contrast to those with $\mathrm{ER}+\geq 10 \%$, have clinicopathological characteristics more similar to those of the ER- negative tumours and did not appear to benefit from endocrine therapy [26]. A retrospective study of early breast cancer including patients with HER2-negative and low hormone (ER and PR) receptor expression tumours reported no clear effect on survival outcomes with the addition of endocrine therapy for patients with low ER/PR (1-9\%) [30]. A further study supported this finding in early breast cancer, reporting that this subgroup behaves clinically like triple-negative $\mathrm{BC}$ in terms of pathological complete response and survival outcomes [31].

All of these results suggest that BC with low ER expression 1-9\% and those with $\leq 1 \%$ of ER may have similar molecular features and clinical prognoses, but differ in terms of efficacy of endocrine therapy compared to patients who have tumours with $\geq 10 \%$ of cells staining positive for ER.

This article is protected by copyright. All rights reserved. 
Whilst the measurement of ER is useful as a predictive biomarker to determine the efficacy of endocrine therapy, the role of PR in predicting response to endocrine therapy for patients with ER+ tumours is controversial. Thus, while it has been reported that benefit from tamoxifen is similar between PR+ and PR-patients [32] and also that PR status does not affect the relative efficacy of AI over tamoxifen [33], other studies report that PR status is an independent predictive indicator of endocrine therapy response and is associated with better overall survival and less relapse $[34,35]$. A recent mechanistic study demonstrated that, in the presence of progesterone, PR guides ER to genomic binding sites associated with more favourable clinical outcomes [36]. Therefore PR positivity can be considered a biomarker of good response to endocrine therapy. Positive PR expression could, in future, be utilised to augment endocrine therapy response by the addition of progesterone to an ER-antagonist. In the meantime, there is still a need to find biomarkers beyond ER and PR status to predict endocrine therapy benefit, due to the complex biology and heterogeneity of luminal BC.

\section{ER-negative BC and benefit from endocrine therapy}

Breast tumours that are ER negative but PR+ by current criteria are difficult to interpret and present a clinical challenge. Typically, patients with ER-/PR+ tumours will be given endocrine therapy. Although some studies do suggest the existence of a PR-positive class within ER-negative tumours, in up to $2-8 \%$ of cases, and that patients with these tumours may benefit from endocrine therapy [34, 37-39], there is controversy over whether the ER$/ \mathrm{PR}+$ phenotype is a real phenomenon. Several recent studies including those from our group have indicated that ER-/PR+ phenotype represents a technical failure, resulting from either false-negative ER or false-positive PR result, at least in the majority of cases [27, 40-42].

This article is protected by copyright. All rights reserved. 
In routine practice we have seen rare cases showing ER-/PR+ phenotype despite repeat staining of both markers. However, the frequency is less than $1 \%$, with 4 cases seen in 9000 breast cancers (unpublished data). As expected these tumours exhibit more aggressive behaviour than the double positive tumours $(\mathrm{ER}+/ \mathrm{PR}+)[37,39,43]$; however, the benefit of endocrine therapy in patients with these rare tumours featuring a genuine ER-/PR+ phenotype remains to be confirmed $[15,27,44,45]$. The very low incidence of the tumours is likely to limit the ability to evaluate the benefit form endocrine therapy in clinical trials.

\section{Gene expression profiling}

Gene expression profiling (GEP) studies over the past two decades have led to the molecular classification of $\mathrm{BC}$ into at least four subtypes. Computational methods have been used to subclassify $\mathrm{BC}$ based on gene expression pattern across clinical samples. The first study identified four subtypes based on expression of ESR1 (the gene encoding ER), ER-related genes and HER2. In this classification system, ER+ tumours comprise the largest class characterised by expression of not only ESR1 and ER-related genes, but also of low molecular weight cytokeratins and other genes characteristic of luminal epithelial cells. This intrinsic subtype was termed luminal class with no further sub-classification [46].

A subsequent study interrogated GEP data obtained from 78 BC cases and seven nonmalignant breast samples, and further defined the intrinsic molecular subtypes of BC. This study classified luminal tumours into 3 subclasses including luminal-A, B and C. Luminal-B and $\mathrm{C}$ were associated with poor prognosis and outcomes compared to luminal-A, which had the highest expression of ER and ER-regulated genes. In contrast to luminal-A and B, luminal-C shared a high expression of genes with HER2 and basal-like subtypes, but with an unknown function [47].

This article is protected by copyright. All rights reserved. 
Subsequent study by the same investigators using hierarchical clustering based on patterns of expression of 534 "intrinsic" genes failed to robustly identify luminal-C, and it subdivided luminal-like BC into luminal-A and luminal-B subtypes. Thus, some of the genes that previously clustered in the luminal-C subtype were clustered in the luminal-B and basal-like [48]. Classification of luminal BC differs between studies, most likely because of the identification and use of distinct intrinsic gene sets for cluster analysis. Most studies using GEP support the sub-classification of luminal tumours into luminal-A and B [6].

Although some luminal-B tumours have a similar signature to the HER2+ subtype, they are discriminated mainly by proliferation-related genes such as MKI67, CCNB1A and MYBL2. Luminal-A disease is a low proliferation phenotype associated with good prognosis and outcome, whereas the proliferative phenotype luminal-B is associated with worse clinical outcome [49]. Luminal-B tumours, though ER+, have a distinct molecular phenotype from the good-prognosis luminal-A subtype. Indeed, luminal-B tumours share many molecular features with ER-negative subtypes. Furthermore, the luminal-B subtype is defined by the expression of genes associated with endocrine therapy resistance such as CCNB1, MKI67, MYBL2, FGFR1 and ZNF703 [49]. However, classification of BC into luminal-A or B does not differentiate tumours according to endocrine therapy response and has not been incorporated into routine clinical practice.

\section{Multigene expression signatures}

Multigene signatures can be valuable as prognostic tools with regards to recurrence and the stratification of risk, but so far studies have not validated their value in predicting benefit from endocrine therapy. Additionally, most of these gene signatures are focused on association with outcome following adjuvant treatment with endocrine therapy with or 
without chemotherapy. Clinically, they have found useful application as biomarkers to pick out patients at high risk of relapse who would benefit most from the addition of chemotherapy prior to their endocrine treatment. However, they have not yet found a role in predicting benefit from endocrine therapy itself.

It is important to investigate the value of multigene signatures in terms of predicting benefit from endocrine treatment, as this may also guide clinical decision making. Table 1 summarises these multigene assays and recommendations for their use in ER+ BC.

Improving upon the current panel of two biomarkers (ER and PR expression) to predict endocrine therapy benefit may in future identify patients who could be spared endocrine therapy and its associated side effects. Alternatively, better biomarkers could be used to identify patients who would benefit from alternative approaches such as CDK4/6 or MTOR inhibition, either alone or in combination with current endocrine therapy.

Specific multigene signatures will now be discussed, with particular reference to their potential utility as predictors of benefit from endocrine therapy.

\section{PAM50 Prosigna}

The PAM50 Prosigna assay was developed to classify breast tumours into one of four intrinsic subtypes (Luminal-A, Luminal-B, HER2-enriched, and Basal-like), based on the expression measurement of 50 genes [50], which were first described in early GEP microarray studies [46-48]. This assay is performed on RNA isolated from FFPE breast tumour tissue and generates a risk of recurrence (ROR) score by weighting the molecular subtype correlations, pathologic tumour size and a subset of proliferation genes $[50,51]$. PAM50 ROR score and intrinsic subtype can identify clinically relevant prognostic 
subgroups of patient with a limited risk of metastasis after endocrine treatment only, for whom chemotherapy can be spared [52]. Additionally, another study reported that PAM50 ROR provided significant additional prognostic information with respect to late distant recurrence (DR)-free survival compared with clinicopathological risk factors [53]. The ASCO guidelines recommend that this assay may be used for patient with ER/PR+, HER2negative (node-negative) early stage $\mathrm{BC}$, in conjunction with other clinicopathologic variables, for predicting outcome and aiding decisions on adjuvant systemic chemotherapy [54].

PAM50 can potentially be utilised as a tool to determine whether extended endocrine therapy is required (beyond 5 years), although the assay has not yet been validated for this purpose. However, PAM50 lacks the ability to predict benefit from endocrine therapy as a first line treatment in early ER+ BC. Moreover, despite the fact that studies have confirmed that the PAM50 score can be used to determine which patients carry a high risk of late DR, this ability is more prognostic than predictive.

\section{Oncotype DX}

The Oncotype DX recurrence score (RS) is based on measuring the mRNA expression levels of 16 cancer related-genes and 5 housekeeping genes from FFPE tissue using the real-time qRT-PCR. This assay was developed for patients with ER+ lymph-node negative BC treated with tamoxifen to predict the risk of DR. The RS classifies patients into three groups: low, intermediate or high risk $[55,56]$. Several reports have shown that the RS assay not only quantifies the likelihood of $\mathrm{BC}$ recurrence in $\mathrm{ER}+$ patients, but also predicts the magnitude of chemotherapy benefit; thus low-risk patients derive minimal, if any, benefit from chemotherapy, while high-risk patients require more aggressive regimens $[55,57,58]$. 
Despite the clinical validation of Oncotype DX, there remain questions as to whether this assay provides more independent prognostic information than can be gained from immunohistochemical assay of known markers such as ER, PR, HER2 and Ki67 (see later section on IHC4 score) [59]. Thus there may be a simpler and cheaper way of obtaining the same prognostic information. However, in favour of Oncotype DX, it is argued that immunohistochemical determination of Ki67 lacks standardisation among laboratories and there is a lack of quality assurance programmes. PAM50 ROR, in comparison to the

Oncotype DX RS, was found to be a more useful prognostic assay for predicting the risk of DR after endocrine treatment in ER+ patients, with better differentiation of intermediate- and higher-risk groups [60]. The Oncotype DX assay can be useful in predicting the benefit from chemotherapy, but is not currently helpful in the clinical setting for predicting the benefit from endocrine therapy for early stage ER+ BC. The St. Gallen Group and ASCO guidelines recommend Oncotype DX RS for newly diagnosed BC patients with ER+/HER2-negative to determine prognosis and aiding decision of chemotherapy [14, 54].

\section{MammaPrint}

The MammaPrint assay measures the expression of 70 genes involved in metastasis, proliferation, tumour stroma and invasion in $\mathrm{BC}$, irrespective of ER status. This assay is a gene microarray-based prognostic score that stratifies patients into low-risk or high-risk groups and utilises fresh tissue that has had its RNA stabilised [61, 62]. Again, like the other gene tests, it is able to demonstrate that only the high-risk ER+ patients could potentially benefit from chemotherapy, whereas low-risk group could be spared this type of treatment [63, 64]. In ER+/PR+ (HER2-negative) patients, this assay was shown to be an independent prognostic marker and it may be integrated into a selection of strategies for patients who are candidates for more aggressive therapeutic approaches [65].

This article is protected by copyright. All rights reserved. 
The clinical utility of MammaPrint was recently confirmed in an international, prospective, randomised trail (MINDACT). The study found that women with early-stage BC, who were at a high clinicopathological risk and a low genomic risk according to MammaPrint for recurrence, could be spared chemotherapy [66]. The ASCO guidelines recommends the use of MammaPrint for patient with ER+/PR+, HER2-negative (node-negative) early stage BC, to guide decisions on adjuvant systemic chemotherapy [67], while St. Gallen group and EGTM do not differentiate between lymph node-negative and lymph node-positive disease $[14,68]$.

The requirement for fresh tissue has limited the clinical uptake of MammaPrint, as this is not always routinely available. The performance, validation and optimisation of MammaPrint using FFPE tissue remains to be validated [69].

\section{Breast cancer index $(B C I)$}

The BCI is an algorithmic combination of a molecular grade index (MGI), consisting of the average expression of five cell cycle (proliferation)-associated genes (BUB1B, CENPA, NEK2, RACGAP1 and RRM2), the HOXB13:IL17BR (H:I) ratio which represents activation of the ER signalling pathway, and 4 housekeeping genes. The BCI assay uses RT-PCR and can be applied to FFPE tissues [70, 71]. The independent prognostic value of BCI in predicting the risk of distant metastasis in ER+/HER2- patients treated with endocrine therapy has been validated in several studies [72-74]. A recent study showed that BCI has more prognostic accuracy than Oncotype DX, indicating that additional subsets of patients with $\mathrm{ER}+\mathrm{BC}$ identified by BCI may be suitable candidates for extended endocrine therapy [75]. The St. Gallen Group and ASCO guidelines recommend that BCI may be used for 
predicting outcome and aiding decision of chemotherapy for patients with ER+/HER2negative $\mathrm{BC}[14,54]$.

\section{EndoPredict (EP)}

The EndoPredict (EP) assay measures the gene expression profiles of eight BC-relevant genes (BIRC5, UBE2C, DHCR7, RBBP8, IL6ST, AZGP1, MGP and STC2) and 3 housekeeping genes in FFPE tissue sections by qRT-PCR in decentralised laboratories. The assay is useful for predicting the likelihood of DR in patients with ER+ HER2- BC treated with endocrine therapy [76]. The combination of EP with the two clinicopathological parameters, tumour size and nodal status, has resulted in an EPclin risk score, which can classify patients into low- and high-risk recurrence groups [77]. A study of 1702 postmenopausal ER+/HER2-negative BC patients from two adjuvant phase III trials (ABCSG6, ABCSG8) showed that both EP and EPclin are useful prognostic tools for the identification of early and late DR following 5 years of adjuvant endocrine therapy [78]. The EPclin assay provides more prognostic information on late DR (10 years) after endocrine therapy than Oncotype DX, partly because of its integration with molecular data and the clinicopathological factors [79]. However, further validation for predicting late recurrence following endocrine therapy is required.

This article is protected by copyright. All rights reserved. 
Table 1: Summary of predictive and prognostics models and recommendations of their use in ER+ BC.

\begin{tabular}{|llllll} 
Assay No. of genes & $\begin{array}{l}\text { Sample } \\
\text { type }\end{array}$ & Platform & Risk category & $\begin{array}{l}\text { Clinical } \\
\text { utility }\end{array}$ & $\begin{array}{l}\text { Prediction of Recommendations } \\
\text { endocrine } \\
\text { therapy } \\
\text { efficacy }\end{array}$
\end{tabular}

\section{Multigene signatures:}

\section{PAM50}

50

Prosigna

FFPE

PCR-based
PAM50
signature on
the
NanoString
platform

Low,

intermediate or

high risk for

DR

platform

FFPE RT-PCR

$\begin{array}{ll}\text { Fresh } & \text { DNA } \\ \text { tissue } & \text { microarray } \\ \text { /FFPE } & \end{array}$

Low or high

risk for DR

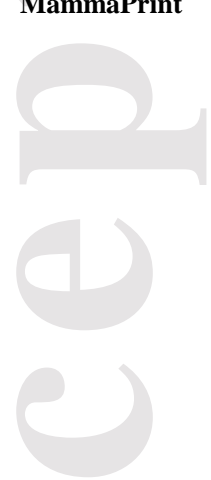

Breast cancer index (BCI)

FFPE

RT-PCR

Low or high risk for DR
Low,

intermediate or high risk for DR

\section{Oncotype DX}

70

/FFPE

\section{Prognostic No}

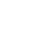
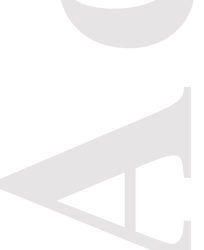

EndoPredict

Prognostic/ No predictive

Prognostic

No

1. ASOC and EGTM for patient with $\mathrm{ER} / \mathrm{PR}+, \mathrm{HER} 2$-negative (node-negative) early stage $\mathrm{BC}$, to guide decisions on adjuvant systemic chemotherapy $[54,68]$. 2. St. Gallen group do not differentiate between lymph node-negative and lymph node-positive disease [14].

1. For patient with ER/PR+, HER2-negative (node-negative) early stage $\mathrm{BC}$, to guide decisions on adjuvant systemic chemotherapy [54].

2. St. Gallen group and EGTM do not differentiate between lymph node-negative and lymph node-positive disease [14, 68]. 


\section{Single biomarkers}

\section{ER}

Level of

FFPE

expression

FFPE

IHC

FFPE

IHC

FISH*

Low or high

Positive or negative

Prognostic

Predictive

Yes

negative

Predictive

Yes

Predictive
ASCO [54], St. Gallen Group [14] and EGTM [68] recommend ER measurement on all newly diagnosed primary invasive $\mathrm{BC}$.

ASCO [54], St. Gallen Group [14] and EGTM [68] recommend HER2 measurement to guide anti-HER2 therapy in patients with early or advanced BC.

St. Gallen Group [14] and EGTM [68] recommend Ki-67 measurement in combination with established clinical and pathological factors for determining prognosis in early BC.

ASCO do not recommend its use because lack of reproducibility and standardisation [54].

*FISH: Fluorescence in situ hybridization. 


\section{IHC surrogates for molecular classification}

With the limited early application of the molecular classes identified using GEP in clinical practice, robust alternatives including IHC biomarkers have been adopted as a surrogate for molecular profiling and prognostic stratification of patients.

Using standard IHC methods, BC can be divided into luminal, HER2-enriched and triplenegative subtypes (using ER, PR and HER2). Means of distinguishing between luminal-A and B subtypes include Ki67 [80] and PR expression to definine luminal-B subtype [5]. Although IHC is considered the standard assay for Ki67 determination, significant variation between centres in assessment of Ki67 expression continues to limit its use in clinical management of $\mathrm{BC}[81,82]$. As a consequence, the St Gallen International Expert Consensus (2013) defined luminal-BC subtypes using IHC as luminal-A (ER+, HER2-, <14\% Ki-67 and $\geq 20 \%$ PR), luminal-B, subdivided into luminal-B HER2-negative (ER+, HER2- and at least one of: Ki-67 $\geq 14 \%$ or PR negative or low), luminal-B HER2-positive (ER+, HER2+ regardless of Ki67 or PR) [14].

In terms of benefit from endocrine therapy, the St Gallen consensus suggested that luminal-A should be treated at relapse with endocrine therapy as it is less responsive to chemotherapy, while luminal-B HER2-negative should be treated with endocrine therapy plus chemotherapy. However, luminal-A tumours could be treated with chemotherapy in addition to endocrine therapy based on risk assessment e.g. large tumour size or patient preference [14]. This demonstrates that there remains uncertainty regarding risk assessment and choice of therapy and more precise molecular classification of $\mathrm{ER}+\mathrm{BC}$ is required in order to better predict benefit from endocrine therapy.

This article is protected by copyright. All rights reserved. 


\section{IHC4 Assay}

IHC4 is a prognostic tool that measures the immunohistochemical level of four key markers ER, PR, HER2 and Ki-67 to predict risk of recurrence for patients treated with endocrine therapy, illustrated in Fig. 1. The IHC4 assay is performed on FFPE tissue and a risk score for recurrence is calculated. The amount of prognostic information of this assay is similar to that provided by Oncotype DX, and has been validated in an independent data set [59]. The main limitation of the IHC4 assay remains the lack of standardised quantification of the biomarkers, particularly with respect to Ki67 [83]. Like the multigene assays, IHC4 is not able to predict which patients will benefit from endocrine therapy, nor pick out those patients with early $\mathrm{BC}$ who would benefit from a combination of endocrine therapy and other targeted treatments.

\section{Mammostrat}

Mammostrat is an IHC based assay that measures the levels of five biomarkers (P53, SLC7A5, NDRG1, CEACAM5 and HTF9C) to provide an assessment of features of tumour biology distinct from hormone receptor status, HER2, or proliferation. This assay applied to FFPE tissues of ER+ BC tamoxifen-treated patients is able to predict the relative risk of recurrence (high, moderate or low) [84] and has been subsequently validated [85-87]. In patients with ER+ HER2- tumours, this assay may be used for determining prognosis and guiding decision making with respect to the use of chemotherapy. There is no data as yet on the clinical utility of the Mammostat assay in predicting benefit from endocrine therapy.

This article is protected by copyright. All rights reserved. 


\section{Nottingham Prognostic Index Plus (NPI+)}

NPI+ is based on the well-established clinicopathological variables used in the traditional NPI but has been refined to integrate tumour biology with these clinicopathological factors. Primary invasive breast carcinoma are initially classified into seven distinct molecular classes using a panel of 10 biomarkers (ER, PR, CK 5/6, CK7/8, EGFR, HER2, HER3, HER4, p53 and Mucin 1) which constitutes three luminal classes characterised by high luminal Ck7/8 and hormone receptor expression [88], the classification is shown in decision tree format in Fig. 2. Luminal-A and Luminal-B classes show high expression of CK7/8, ER, HER3 and HER4 but are separated by lower levels of PR expression in Luminal-B. Luminal-N class shows differential expression of HER3 and HER4. The classes are followed by a second stage of stratification to incorporate clinicopathological variables, resulting in distinct prognostic groups within each molecular class [88].

Using the NPI+ formulae, the NPI+ distinctive classes of BC achieved an improved patient outcome stratification compared to the traditional NPI. Recently, the reproducibility and prognostic value of this tool was validated in an independent cohort of primary BC [89], and was suggested to be a useful tool in predicting the risk of metastases in primary BC [90]. However, despite the utility of this assay, it has limited literature to determine its ability to predict benefit form endocrine therapy.

\section{Are the above discussed assays useful in predicting benefit from endocrine therapy?}

There is increasing concern that all the assays discussed above are not sufficient to predict which patients with early $\mathrm{BC}$ will benefit from endocrine therapy, as their prognostic values are in predicting risk of recurrence or metastases. Furthermore, despite the fact that IHC4 has clinical validity for selecting women with $\mathrm{ER}+\mathrm{BC}$ who might be spared extended endocrine 
therapy, there remains a clinical need for further research to establish new biomarkers to predict the benefit of endocrine therapy in early stage ER+ BC.

The side effects of prolonged endocrine therapy are not inconsiderable. Debilitating nonagent specific side effects of endocrine therapy that impair quality of life include hot flushes, fatigue and myalgia. Additionally, there are potentially serious side effects including bone demineralisation and osteoporosis (with AIs), and life-threatening toxicities such as venous thromboembolism (with tamoxifen). A biomarker assay that could pick out people with BC that would not benefit from endocrine therapy could spare people these risks and improve quality of life.

On the other hand, assays such as those discussed above can pick out patients at higher risk of relapse, who should therefore be given more intense treatment with chemotherapy. In the future, biomarkers of endocrine therapy benefit may also pick out those at higher risk who would benefit from combinations of endocrine therapy with other targeted therapies such as MTOR or CDK4/6 inhibitors.

\section{Conclusion and future directions}

At present, there are no biomarkers that can be used to reliably predict which patients will benefit from endocrine therapy. Prediction of benefit from endocrine therapy is important in order to treat only those that have a good chance to respond, and spare non-responders from the side effects of additional hormonal treatment.

Despite the clear advancement in multigene signatures in molecular classification or risk stratification of $\mathrm{BC}$, none of these assays have found clinical utility in assessing endocrine therapy benefit in early stage ER+ BC, prior to treatment. Future studies that aim to identify 
new predictive biomarkers may integrate detailed clinicopathological and histopathological data from large cohorts with full clinical annotation and long-term follow-up, and use multivariate model testing to establish the independence of potential biomarkers in addition to ER and PR.

To identify new molecular biomarkers predictive of benefit from endocrine therapy, a retrospective study could be conducted using clinically annotated gene expression data from large cohorts, comparing clinical outcome in those treated only with endocrine therapy. This could then be integrated with protein expression data to produce a combined multigene signature and IHC score specific for predicting benefit from endocrine therapy.

\section{References:}

1. Rondón-Lagos, M., et al., Tamoxifen Resistance: Emerging Molecular Targets. International Journal of Molecular Sciences, 2016. 17(8): p. 1357.

2. Anderson, P.I.E.C.D.S.A.-S.D.N., et al., Pathology reporting of breast disease in surgical excision specimens incorporating the dataset for histological reporting of breast cancer. 2016. 2.

3. Olivotto, I.A., et al., Time to stop progesterone receptor testing in breast cancer management. J Clin Oncol, 2004. 22(9): p. 1769-70.

4. Purdie, C.A., et al., Progesterone receptor expression is an independent prognostic variable in early breast cancer: a population-based study. British Journal of Cancer, 2014. 110(3): p. 565-572.

5. Prat, A., et al., Prognostic significance of progesterone receptor-positive tumor cells within immunohistochemically defined luminal A breast cancer. J Clin Oncol, 2013. 31(2): p. 203-9.

6. Abd El-Rehim, D.M., et al., High-throughput protein expression analysis using tissue microarray technology of a large well-characterised series identifies biologically distinct classes of breast cancer confirming recent cDNA expression analyses. Int J Cancer, 2005. 116(3): p. 340-50.

7. Creighton, C.J., The molecular profile of luminal B breast cancer. Biologics : Targets \& Therapy, 2012. 6: p. 289-297.

8. Ellis, M.J., et al., Outcome prediction for estrogen receptor-positive breast cancer based on postneoadjuvant endocrine therapy tumor characteristics. J Natl Cancer Inst, 2008. 100(19): p. $1380-8$.

9. Miller, W.R., et al., Aromatase inhibitors: are there differences between steroidal and nonsteroidal aromatase inhibitors and do they matter? Oncologist, 2008. 13(8): p. 829-37.

10. Burstein, H.J., et al., American Society of Clinical Oncology Clinical Practice Guideline: Update on Adjuvant Endocrine Therapy for Women With Hormone Receptor-Positive Breast Cancer. Journal of Clinical Oncology, 2010. 28(23): p. 3784-3796.

This article is protected by copyright. All rights reserved. 
11. Robertson, J.F.R., et al., Fulvestrant $500 \mathrm{mg}$ versus anastrozole $1 \mathrm{mg}$ for hormone receptorpositive advanced breast cancer (FALCON): an international, randomised, double-blind, phase 3 trial. The Lancet. 388(10063): p. 2997-3005.

12. Agency, E.M. Fulvestrant summary of product characteristics. 2017 02/10/2017 [cited 2017 01 Dec 2017].

13. Administration, U.S.F.a.D. Fulvestrant Highlights of Prescribing Information. 2017 [cited 201701 Dec 2017].

14. Goldhirsch, A., et al., Personalizing the treatment of women with early breast cancer: highlights of the St Gallen International Expert Consensus on the Primary Therapy of Early Breast Cancer 2013. Ann Oncol, 2013. 24(9): p. 2206-23.

15. Early Breast Cancer Trialists' Collaborative, G., Relevance of breast cancer hormone receptors and other factors to the efficacy of adjuvant tamoxifen: patient-level meta-analysis of randomised trials. The Lancet, 2011. 378(9793): p. 771-784.

16. Freedman, O.C., et al., Adjuvant endocrine therapy for early breast cancer: a systematic review of the evidence for the 2014 Cancer Care Ontario systemic therapy guideline. Current Oncology, 2015. 22(Suppl 1): p. S95-S113.

17. Cardoso, F., et al., ESO-ESMO 2nd international consensus guidelines for advanced breast cancer (ABC2). Breast, 2014. 23(5): p. 489-502.

18. Mouridsen, H., et al., Phase III study of letrozole versus tamoxifen as first-line therapy of advanced breast cancer in postmenopausal women: analysis of survival and update of efficacy from the International Letrozole Breast Cancer Group. J Clin Oncol, 2003. 21.

19. Regan, M.M., et al., Absolute Benefit of Adjuvant Endocrine Therapies for Premenopausal Women With Hormone Receptor-Positive, Human Epidermal Growth Factor Receptor 2Negative Early Breast Cancer: TEXT and SOFT Trials. Journal of Clinical Oncology, 2016. 34(19): p. 2221-2231.

20. Yu, Q., et al., Requirement for CDK4 kinase function in breast cancer. Cancer Cell, 2006. 9(1): p. 23-32.

21. Choi, Y.J., et al., The requirement for cyclin D function in tumor maintenance. Cancer Cell, 2012. 22(4): p. 438-51.

22. de Groot, A.F., C.J. Kuijpers, and J.R. Kroep, CDK4/6 inhibition in early and metastatic breast cancer: A review. Cancer Treatment Reviews, 2017. 60: p. 130-138.

23. Administration., U.S.F.a.D. FDA approves Ibrance for postmenopausal women with advanced breast cancer. 2017 31/3/2017 [cited 201828 Feb].

24. Administration, U.S.F.a.D. Ribociclib 2017 14/03/2018 [cited 201828 Feb].

25. Company, E.L.a. A Randomized, Open-Label, Phase 3 Study of Abemaciclib Combined With Standard Adjuvant Endocrine Therapy Versus Standard Adjuvant Endocrine Therapy Alone in Patients With High Risk, Node Positive, Early Stage, Hormone Receptor Positive, Human Epidermal Receptor 2 Negative, Breast Cancer. 201726 Feb 2018 [cited 201828 Feb].

26. Yi, M., et al., Which threshold for ER positivity? a retrospective study based on 9639 patients. Annals of Oncology, 2014. 25(5): p. 1004-1011.

27. Hammond, M.E.H., et al., American Society of Clinical Oncology/College of American Pathologists Guideline Recommendations for Immunohistochemical Testing of Estrogen and Progesterone Receptors in Breast Cancer. Journal of Clinical Oncology, 2010. 28(16): p. 27842795.

28. Iwamoto, T., et al., Estrogen receptor (ER) MRNA and ER-related gene expression in breast cancers that are 1\% to 10\% ER-positive by immunohistochemistry. J Clin Oncol, 2012. 30(7): p. 729-34.

29. Deyarmin, B., et al., Effect of ASCO/CAP guidelines for determining ER status on molecular subtype. Ann Surg Oncol, 2013. 20(1): p. 87-93.

30. Balduzzi, A., et al., Survival Outcomes in Breast Cancer Patients With Low Estrogen/Progesterone Receptor Expression. Clinical Breast Cancer. 14(4): p. 258-264.

This article is protected by copyright. All rights reserved. 
31. Fujii, T., et al., Revisiting the definition of estrogen receptor positivity in HER2-negative primary breast cancer. Ann Oncol, 2017. 28(10): p. 2420-2428.

32. Group, E.B.C.T.C., Effects of chemotherapy and hormonal therapy for early breast cancer on recurrence and 15-year survival: an overview of the randomised trials. The Lancet, 2005. 365(9472): p. 1687-1717.

33. Viale, G., et al., Prognostic and predictive value of centrally reviewed expression of estrogen and progesterone receptors in a randomized trial comparing letrozole and tamoxifen adjuvant therapy for postmenopausal early breast cancer: BIG 1-98. J Clin Oncol, 2007. 25(25): p. 3846-52.

34. Bardou, V.J., et al., Progesterone receptor status significantly improves outcome prediction over estrogen receptor status alone for adjuvant endocrine therapy in two large breast cancer databases. J Clin Oncol, 2003. 21(10): p. 1973-9.

35. Stendahl, M., et al., High progesterone receptor expression correlates to the effect of adjuvant tamoxifen in premenopausal breast cancer patients. Clin Cancer Res, 2006. 12(15): p. 4614-8.

36. Mohammed, H., et al., Progesterone receptor modulates ERalpha action in breast cancer. Nature, 2015. 523(7560): p. 313-7.

37. Rakha, E.A., et al., Biologic and clinical characteristics of breast cancer with single hormone receptor positive phenotype. J Clin Oncol, 2007. 25(30): p. 4772-8.

38. Yang, L.-H., et al., Survival Benefit of Tamoxifen in Estrogen Receptor-Negative and Progesterone Receptor-Positive Low Grade Breast Cancer Patients. Journal of Breast Cancer, 2012. 15(3): p. 288-295.

39. $\mathrm{Ng}, \mathrm{C} . \mathrm{H}$. , et al., The estrogen receptor negative-progesterone receptor positive breast carcinoma is a biological entity and not a technical artifact. Asian Pac J Cancer Prev, 2012. 13(4): p. 1111-3.

40. Nadji, M., et al., Immunohistochemistry of estrogen and progesterone receptors reconsidered: experience with 5,993 breast cancers. Am J Clin Pathol, 2005. 123(1): p. 21-7.

41. Maeyer, L.D., et al., Does Estrogen Receptor-Negative/Progesterone Receptor-Positive Breast Carcinoma Exist? Journal of Clinical Oncology, 2008. 26(2): p. 335-336.

42. Hefti, M.M., et al., Estrogen receptor negative/progesterone receptor positive breast cancer is not a reproducible subtype. Breast Cancer Res, 2013. 15(4): p. R68.

43. Chan, M., et al., Outcomes of Estrogen Receptor Negative and Progesterone Receptor Positive Breast Cancer. PLoS ONE, 2015. 10(7): p. e0132449.

44. Yu, K.D., et al., Molecular essence and endocrine responsiveness of estrogen receptornegative, progesterone receptor-positive, and HER2-negative breast cancer. BMC Med, 2015. 13: p. 254.

45. Yu, K.D., et al., Breast cancer patients with estrogen receptor-negative/progesterone receptor-positive tumors: being younger and getting less benefit from adjuvant tamoxifen treatment. J Cancer Res Clin Oncol, 2008. 134(12): p. 1347-54.

46. Perou, C.M., et al., Molecular portraits of human breast tumours. Nature, 2000. 406(6797): p. 747-52.

47. Sorlie, T., et al., Gene expression patterns of breast carcinomas distinguish tumor subclasses with clinical implications. Proc Natl Acad Sci U S A, 2001. 98(19): p. 10869-74.

48. Sorlie, T., et al., Repeated observation of breast tumor subtypes in independent gene expression data sets. Proc Natl Acad Sci U S A, 2003. 100(14): p. 8418-23.

49. Zhang, M.H., et al., Estrogen receptor-positive breast cancer molecular signatures and therapeutic potentials (Review). Biomedical Reports, 2014. 2(1): p. 41-52.

50. Parker, J.S., et al., Supervised risk predictor of breast cancer based on intrinsic subtypes. J Clin Oncol, 2009. 27(8): p. 1160-7.

This article is protected by copyright. All rights reserved. 
51. Nielsen, T.O., et al., A comparison of PAM50 intrinsic subtyping with immunohistochemistry and clinical prognostic factors in tamoxifen-treated estrogen receptor-positive breast cancer. Clin Cancer Res, 2010. 16(21): p. 5222-32.

52. Gnant, M., et al., Identifying clinically relevant prognostic subgroups of postmenopausal women with node-positive hormone receptor-positive early-stage breast cancer treated with endocrine therapy: a combined analysis of ABCSG-8 and ATAC using the PAM50 risk of recurrence score and intrinsic subtype. Ann Oncol, 2015. 26(8): p. 1685-91.

53. Filipits, M., et al., The PAM50 risk-of-recurrence score predicts risk for late distant recurrence after endocrine therapy in postmenopausal women with endocrine-responsive early breast cancer. Clin Cancer Res, 2014. 20(5): p. 1298-305.

54. Harris, L.N., et al., Use of Biomarkers to Guide Decisions on Adjuvant Systemic Therapy for Women With Early-Stage Invasive Breast Cancer: American Society of Clinical Oncology Clinical Practice Guideline. Journal of Clinical Oncology, 2016. 34(10): p. 1134-1150.

55. Paik, S., Development and clinical utility of a 21-gene recurrence score prognostic assay in patients with early breast cancer treated with tamoxifen. Oncologist, 2007. 12(6): p. 631-5.

56. Sparano, J.A. and S. Paik, Development of the 21-gene assay and its application in clinical practice and clinical trials. J Clin Oncol, 2008. 26(5): p. 721-8.

57. Paik, S., et al., Gene expression and benefit of chemotherapy in women with node-negative, estrogen receptor-positive breast cancer. J Clin Oncol, 2006. 24(23): p. 3726-34.

58. Goldstein, L.J., et al., Prognostic utility of the 21-gene assay in hormone receptor-positive operable breast cancer compared with classical clinicopathologic features. J Clin Oncol, 2008. 26(25): p. 4063-71.

59. Cuzick, J., et al., Prognostic value of a combined estrogen receptor, progesterone receptor, Ki67, and human epidermal growth factor receptor 2 immunohistochemical score and comparison with the Genomic Health recurrence score in early breast cancer. J Clin Oncol, 2011. 29(32): p. 4273-8.

60. Dowsett, M., et al., Comparison of PAM50 risk of recurrence score with oncotype DX and IHC4 for predicting risk of distant recurrence after endocrine therapy. J Clin Oncol, 2013. 31(22): p. 2783-90.

61. Buyse, M., et al., Validation and clinical utility of a 70-gene prognostic signature for women with node-negative breast cancer. J Natl Cancer Inst, 2006. 98(17): p. 1183-92.

62. van de Vijver, M.J., et al., A gene-expression signature as a predictor of survival in breast cancer. N Engl J Med, 2002. 347(25): p. 1999-2009.

63. Knauer, M., et al., The predictive value of the 70-gene signature for adjuvant chemotherapy in early breast cancer. Breast Cancer Res Treat, 2010. 120(3): p. 655-61.

64. Drukker, C.A., et al., A prospective evaluation of a breast cancer prognosis signature in the observational RASTER study. Int J Cancer, 2013. 133(4): p. 929-36.

65. Saghatchian, M., et al., Additional prognostic value of the 70-gene signature (MammaPrint((R))) among breast cancer patients with 4-9 positive lymph nodes. Breast, 2013. 22(5): p. 682-90.

66. Cardoso, F., et al., 70-Gene Signature as an Aid to Treatment Decisions in Early-Stage Breast Cancer. New England Journal of Medicine, 2016. 375(8): p. 717-729.

67. Krop, I., et al., Use of Biomarkers to Guide Decisions on Adjuvant Systemic Therapy for Women With Early-Stage Invasive Breast Cancer: American Society of Clinical Oncology Clinical Practice Guideline Focused Update. J Clin Oncol, 2017. 35(24): p. 2838-2847.

68. Duffy, M.J., et al., Clinical use of biomarkers in breast cancer: Updated guidelines from the European Group on Tumor Markers (EGTM). Eur J Cancer, 2017. 75: p. 284-298.

69. Sapino, A., et al., MammaPrint molecular diagnostics on formalin-fixed, paraffin-embedded tissue. J Mol Diagn, 2014. 16(2): p. 190-7.

70. Ma, X.J., et al., A five-gene molecular grade index and HOXB13:IL17BR are complementary prognostic factors in early stage breast cancer. Clin Cancer Res, 2008. 14(9): p. 2601-8.

This article is protected by copyright. All rights reserved. 
71. Zhang, Y., et al., Breast cancer index identifies early-stage estrogen receptor-positive breast cancer patients at risk for early-and late-distant recurrence. Clin Cancer Res, 2013. 19(15): p. 4196-205.

72. Sgroi, D.C., et al., Prediction of late distant recurrence in patients with oestrogen-receptorpositive breast cancer: a prospective comparison of the breast-cancer index (BCI) assay, 21gene recurrence score, and IHC4 in the TransATAC study population. Lancet Oncol, 2013. 14(11): p. 1067-76.

73. Sanft, T., et al., Prospective assessment of the decision-making impact of the Breast Cancer Index in recommending extended adjuvant endocrine therapy for patients with early-stage ER-positive breast cancer. Breast Cancer Res Treat, 2015. 154(3): p. 533-41.

74. Sgroi, D.C., et al., Assessment of the prognostic and predictive utility of the Breast Cancer Index (BCI): an NCIC CTG MA.14 study. Breast Cancer Res, 2016. 18(1): p. 1.

75. Sestak, l., et al., Cross-Stratification and Differential Risk by Breast Cancer Index and Recurrence Score in Women with Hormone Receptor-Positive Lymph Node-Negative EarlyStage Breast Cancer. Clin Cancer Res, 2016. 22(20): p. 5043-5048.

76. Denkert, C., et al., Decentral gene expression analysis for ER+/Her2- breast cancer: results of a proficiency testing program for the EndoPredict assay. Virchows Arch, 2012. 460(3): p. 2519.

77. Filipits, M., et al., A new molecular predictor of distant recurrence in ER-positive, HER2negative breast cancer adds independent information to conventional clinical risk factors. Clin Cancer Res, 2011. 17(18): p. 6012-20.

78. Dubsky, P., et al., The EndoPredict score provides prognostic information on late distant metastases in ER+/HER2- breast cancer patients. Br J Cancer, 2013. 109(12): p. 2959-64.

79. Buus, R., et al., Comparison of EndoPredict and EPclin With Oncotype DX Recurrence Score for Prediction of Risk of Distant Recurrence After Endocrine Therapy. J Natl Cancer Inst, 2016. 108(11).

80. Cheang, M.C., et al., Ki67 index, HER2 status, and prognosis of patients with luminal B breast cancer. J Natl Cancer Inst, 2009. 101(10): p. 736-50.

81. Dowsett, M., et al., Assessment of Ki67 in Breast Cancer: Recommendations from the International Ki67 in Breast Cancer Working Group. JNCI Journal of the National Cancer Institute, 2011. 103(22): p. 1656-1664.

82. Polley, M.-Y.C., et al., An International Ki67 Reproducibility Study. JNCI Journal of the National Cancer Institute, 2013. 105(24): p. 1897-1906.

83. Bartlett, J.M.S., et al., Validation of the IHC4 Breast Cancer Prognostic Algorithm Using Multiple Approaches on the Multinational TEAM Clinical Trial. Archives of Pathology \& Laboratory Medicine, 2016. 140(1): p. 66-74.

84. Ring, B.Z., et al., Novel Prognostic Immunohistochemical Biomarker Panel for Estrogen Receptor-Positive Breast Cancer. Journal of Clinical Oncology, 2006. 24(19): p. 3039-3047.

85. Ross, D.T., et al., Chemosensitivity and Stratification by a Five Monoclonal Antibody Immunohistochemistry Test in the NSABP B14 and B20 Trials. Clinical Cancer Research, 2008. 14(20): p. 6602-6609.

86. Bartlett, J.M.S., et al., Mammostrat $\left({ }^{\circledR}\right)$ as a tool to stratify breast cancer patients at risk of recurrence during endocrine therapy. Breast Cancer Research : BCR, 2010. 12(4): p. R47-R47.

87. Bartlett, J.M.S., et al., Mammostrat As an Immunohistochemical Multigene Assay for Prediction of Early Relapse Risk in the Tamoxifen Versus Exemestane Adjuvant Multicenter Trial Pathology Study. Journal of Clinical Oncology, 2012. 30(36): p. 4477-4484.

88. Rakha, E.A., et al., Nottingham Prognostic Index Plus (NPI+): a modern clinical decision making tool in breast cancer. Br J Cancer, 2014. 110(7): p. 1688-1697.

89. Green, A.R., et al., Nottingham Prognostic Index Plus: Validation of a clinical decision making tool in breast cancer in an independent series. The Journal of Pathology: Clinical Research, 2016. 2(1): p. 32-40.

This article is protected by copyright. All rights reserved. 
90. Green, A.R., et al., Nottingham prognostic index plus (NPI+) predicts risk of distant metastases in primary breast cancer. Breast Cancer Research and Treatment, 2016. 157(1): p. 65-75.

\section{Figures legend:}

Figure 1: Illustration of IHC4 and the corresponding risk score that combines the IHC4 score and the clinical score providing prognostic information on the risk of DR. The IHC4 score is based on the assessment of four key proteins ER, PR, HER2 and Ki-67. The clinical score is based on the evaluation of clinical parameters such as nodal status, tumour size, age, grade and endocrine treatment.

Figure 2: Illustration of NPI+ assay and the use of panel of biomarkers to classify BC into seven distinct molecular classes.

This article is protected by copyright. All rights reserved. 


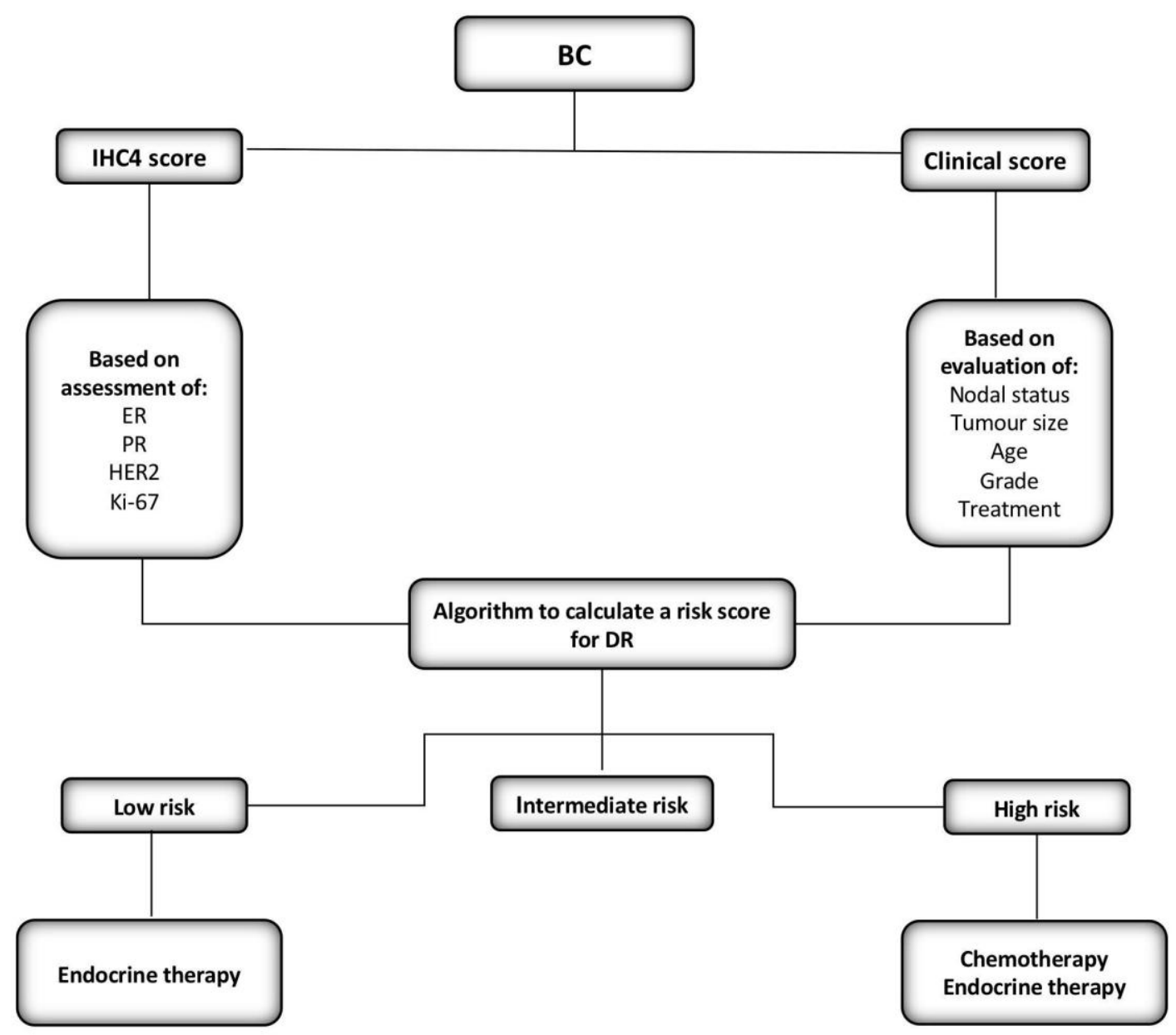

This article is protected by copyright. All rights reserved. 


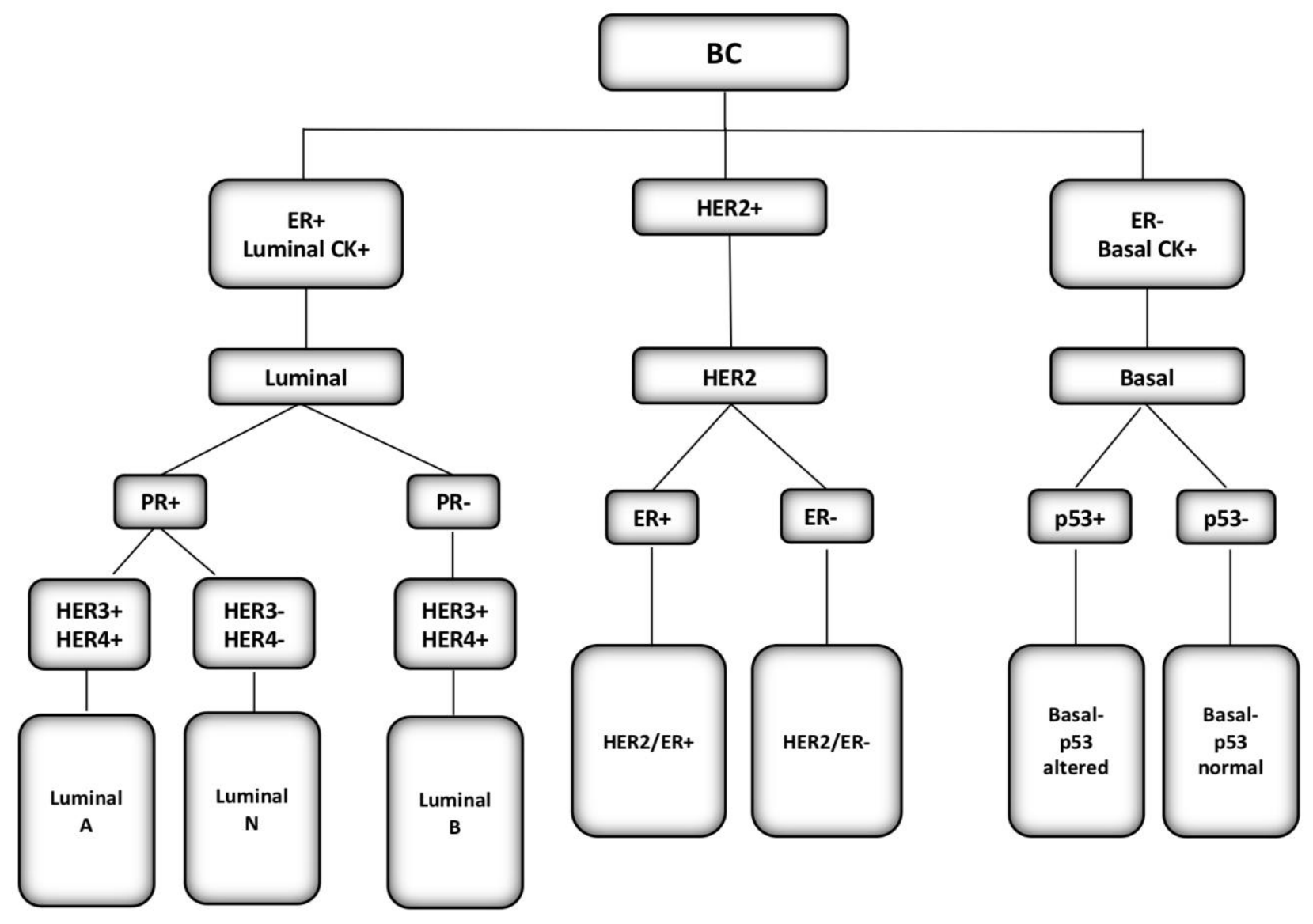

This article is protected by copyright. All rights reserved. 